\title{
Ciclos del viento como condicionante de diseño bioclimático y urbano para Ciudad Valles, S.L.P.
}

\section{Wind cycle as a bioclimatic and urban design conditioner for Ciudad Valles, S.L.P.}

ZAPATA-PADILLA, Néstor Juan*†, PEDRAZA-GÓMEZ, Carlos, BOJÓRQUEZ-VARGAS, Alma Rafaela, HERNÁNDEZ-GONZÁLES, Gisela Beatriz

Universidad Autónoma de San Luis Potosí, Unidad Académica Multidisciplinaria Zona Huasteca Nombre, calle Romualdo del Campo No. 501, Frac. Rafael Curiel, Ciudad Valles, S.L.P., México, C.P. 79060,

ID $1^{\text {er }}$ Autor: Néstor Juan, Zapata-Padilla / ORC ID: 0000-0003-3367-3589, CVU CONACYT ID: 898332

ID $1^{\text {er }}$ Coautor: Carlos, Pedraza-Gómez / ORC ID: 0000-0003-2308-2857, CVU CONACYT ID: 334939

ID $2^{\text {do }}$ Coautor: Alma Rafaela, Bojórquez-Vargas / ORC ID: 0000-0001-9959-5320, CVU CONACYT ID: 162577

ID $3^{\text {er }}$ Coautor: Gisela Beatriz, Hernández-González / ORC ID: 0000-0002-7473-9583, CVU CONACYT ID: 816192

DOI: $10.35429 / J C E .2019 .8 .3 .10 .19$

Recibido 03 de Marzo, 2019; Aceptado 30 Junio, 2019

\section{Resumen}

Objetivo: Identificar y analizar la procedencia del viento en las distintas épocas del año y los diferentes periodos del día para la zona urbana de Ciudad Valles, San Luis Potosí, México, cuyas temperaturas máximas oficialmente rebasan $\operatorname{los} 45^{\circ} \mathrm{C}$. Establecer bases del urbanismo eólico y arquitectura sustentable para adecuar formal $\mathrm{y}$ funcionalmente las obstrucciones y causes del viento en las áreas de tránsito peatonal y vivienda. Metodología: Enfoque descriptivo y cuantitativo. Revisión de fuentes documentales, procesamiento y análisis de información estadística de CONAGUA con apoyo de software Excel, aprendizaje y manejo de software WRPLOT View para la elaboración de gráficas de rosas de los vientos. Contribución: Fortalecer y respaldar la toma de decisiones para desarrollar proyectos, programas y mejoramiento de espacios urbano arquitectónicos, con una visión bioclimática y sustentable. Esclarecer a través de gráficas de rosas de los vientos la dirección de estos en la zona urbana de Ciudad Valles, S.L.P.

Urbanismo eólico, Rosas de los vientos Ciudad Valles

\begin{abstract}
Objective: Identify and analyze the wind's origin for seasons and periods of the day in the urban area of Ciudad Valles, San Luis Potosí, Mexico, officially with temperatures higher $\tan 113^{\circ} \mathrm{F}$. Establish the bases of wind urban planning and sustainable architecture to formally and functionally adapt the obstructions and wind's guides in pedestrian traffic spaces and living place. Methodology: Descriptive and quantitative approach. Review of documentary sources, processing and analysis of statistical information CONAGUA with Excel software support, learning in the management of software WRPLOT See for the elaboration of graphs of roses of the winds. Contribution: Strengthen and support decisions about project development, programs and improvement for urban spaces, with a bioclimatic and sustainable vision. Clarify by graphs and compass roses the wind's direction in the urban area of Ciudad Valles, S.L.P.
\end{abstract}

Wind urbanism, Compass rose of Ciudad Valles

Citación: ZAPATA-PADILLA, Néstor Juan, PEDRAZA-GÓMEZ, Carlos, BOJÓRQUEZ-VARGAS, Alma Rafaela, HERNÁNDEZ-GONZÁLES, Gisela Beatriz. Ciclos del viento como condicionante de diseño bioclimático y urbano para Ciudad Valles, S.L.P. Revista de Ingeniería Civil. 2019. 3-8: 10-19

\footnotetext{
* Correspondencia del Autor (correo electrónico: nestor.zapata@uaslp.mx)

$\dagger$ Investigador contribuyendo como primer autor.
} 


\section{Introducción}

Actualmente; en el siglo XXI; el concepto de proyectar una ciudad como tal, difícilmente aplica, salvo en los casos extraordinarios de los emiratos árabes, por lo que el urbanismo, como un conjunto de disciplinas, se dedica más bien a la planeación del desarrollo y expansión de una mancha urbana ya establecida en relación con el crecimiento y actividades de sus habitantes, además de la intervención en ciertas zonas para mejorar las condiciones de vida respecto de los mismos temas. Esto provoca que con el paso del tiempo se deje de considerar el análisis climatológico del sitio para el diseño bioclimático de ciudades, de igual forma se limita la interpretación y el estudio de la relación que existe entre el medio físico y urbano arquitectónico; aunado a ello; los materiales de construcción modernos obedecen más bien a los principios tecnológicos, económicos e industriales más que a la adaptación y sinergia con el medio ambiente donde se desarrolla la ciudad y la arquitectura.

Por estos motivos el urbanismo sustentable, concebido, apropiado y justificado por el contexto físico, el cual tiene la intención de optimizar los recursos naturales en pro del beneficio humano, es cada vez menos utilizado.

Para desarrollar el proyecto de investigación Eficiencia energética y confort térmico para la persona en las tipologías de vivienda familiar para la región cálida subhúmeda, Ciudad Valles, San Luis Potosí. (1ra etapa: diagnóstico) es necesario estudiar el contexto meso climático de Ciudad Valles, San Luis Potosí, México, de este modo se identifica que la bibliografía existente no es satisfactoria para obtener información climática detallada para el municipio, específicamente la dirección del viento y la humedad relativa.

Para este escrito únicamente se describe el trabajo que se realiza para la observación del viento, dejando para otro documento la observación de los valores para la humedad relativa, la radiación solar y la temperatura. Este análisis se respalda en la información estadística del municipio llamada normales climatológicas, información de acceso libre por medio del Servicio Meteorológico Nacional que depende de la Comisión Nacional del Agua; organismo Público; ajeno a la Secretaría de Medio Ambiente y Recursos Naturales.
El cual cuenta para el año 2018 con 188 estaciones meteorológicas automáticas; denominadas EMAS; distribuidas por todo el territorio nacional, además de los equipos de las distintas dependencias nacionales como la Comisión federal de electricidad; CFE; secretaria de marina; SEMAR; con los que colaboran desde hace más de treinta años $\mathrm{y}$ suman en conjunto más de tres mil puntos destinados a la medición y registro de datos climatológicos en el territorio nacional, incluyendo el territorio marítimo.

En la búsqueda de información climatológica, específicamente la dirección del viento para Ciudad Valles; se encontraron fuentes de información importantes para el desarrollo urbano municipal, las cuales no describen detalladamente esta variable, tal es el caso del Anuario estadístico del estado de San Luis Potosí de 1988; Anuario estadístico y geográfico de San Luis Potosí 2017; Anuario estadístico y geográfico por entidad federativa 2016; Periódico oficial del Gobierno del Estado Libre y Soberano de San Luis Potosí de 1999; Plan de Desarrollo municipal 2012 - 2015; Plan del Centro de población estratégico San Luis Potosí Soledad de Graciano Sánchez del año 2003; Plan Estatal de Desarrollo urbano de San Luis Potosí 2012-2030; Plan Municipal de Desarrollo de Ciudad Valles, San Luis Potosí 2004 - 2006; Prontuario de información geográfica municipal de los Estados Unidos Mexicanos del año 2009; y para el año 2018; entre otros.

De las fuentes que se revisan, se enuncia la dirección del viento para Ciudad Valles únicamente en el Periódico oficial del Gobierno del estado libre y soberano de San Luis Potosí de 1999 donde describe en su inciso de climatología dos punto siete, lo siguiente; “...considerando que los vientos dominantes proceden del noroeste presentando velocidades moderadas."; sin embargo es de interés de la investigación conocer a detalle el comportamiento del viento en la ciudad, por lo que en general las fuentes no son lo suficientemente útiles.

La información que se trabaja para este artículo se obtiene en formato digital vía correo electrónico, en respuesta de un oficio dirigido al Director de la dependencia en base a los procedimientos de solicitud de Información Pública entregado en las oficinas regionales de la CONAGUA instaladas en la ciudad. 
Esta información se recibe en archivos separados, con los cuales se logra producir un concentrado de datos en Excel para las distintas observaciones, el cual se procesa de tal forma que pueda utilizarse en el software WRPLOT view y así realizar las rosas de los vientos mensuales y anuales para la ciudad. Se produce también las gráficas que corresponden a las distintas épocas del año; primavera, verano, otoño e invierno; además, que por cada época se definen también rosas de vientos según el periodo del día; mañana, tarde, noche y madrugada; en horarios de 00:00-6:00h para madrugada, de 6:00-12:00h para mañana, de 12:00-18:00h para tarde y para la noche un horario de 18:00 a 00:00h. Con los datos procesados y las gráficas hechas se logra identificar claramente la dirección de donde procede el viento para Ciudad Valles, San Luis Potosí, México.

\section{Objetivo}

Identificar y analizar la procedencia del viento en las distintas épocas del año y los diferentes periodos del día para la zona urbana de Ciudad Valles, San Luis Potosí, México, cuyas temperaturas máximas oficialmente rebasan los $45^{\circ} \mathrm{C}$. Establecer bases del urbanismo eólico y arquitectura sustentable para adecuar formal y funcionalmente las obstrucciones y causes del viento en las áreas de tránsito peatonal y vivienda.

\section{Objetivos específicos}

Además de consultar la literatura respecto del tema, se solicita a la CONAGUA, conforme a los procedimientos oficiales de acceso a la información, los datos estadísticos que registra la EMA de Ciudad Valles; al recibir la información, se procesa en Excel para analizar, obtener y organizar los resultados de la velocidad en $\mathrm{m} / \mathrm{s}$ y dirección del viento en grados, posteriormente es necesario aprender a utilizar el programa WRPLOT view para auxiliarse de él y generar los gráficos de rosas de los vientos.

\section{Justificación}

Conocer y definir el comportamiento de los vientos para la ciudad es de vital importancia para establecer las bases del urbanismo eólico y arquitectura sustentable en la ciudad; es importante adecuar formalmente.
Así como funcionalmente las obstrucciones del viento particularmente en las áreas de tránsito peatonal y vivienda; esto para las distintas épocas del año, así como para los distintos periodos del día.

La intención de este análisis es fortalecer y respaldar la toma de decisiones en la elaboración de proyectos de creación y mejoramiento de espacios urbanos peatonales considerando por ejemplo sustituir el concreto por vegetación, optimizar la densidad edificada y aumentar las áreas arbóreas, además tener en cuenta la correcta ubicación de ventanas según la procedencia del viento, así mismo implementar sistemas de ventilación natural para la vivienda en la ciudad; todo esto pensando en el bienestar y confort de la persona sin olvidar el sector turístico de la región al mejorar las condiciones del espacio urbano.

\section{Planteamiento del problema}

La problemática más relevante para la presente investigación es que los distintos programas municipales de desarrollo urbano carecen de información climática detallada, importante información que justifica el origen e implementación de proyectos urbanos sustentables. Al realizar la búsqueda de información climatológica para el municipio de Ciudad Valles, San Luis Potosí, México, sobre todo en medios como la internet y documentos digitales del municipio, difícilmente se lograr esclarecer la dirección del viento como para realizar un análisis profundo; en este caso la rosa de los vientos en sus distintas épocas del año y periodos del día.

\section{Hipótesis}

Dada la lectura, la revisión y el análisis de las distintas fuentes de información que se consultan para conocer los antecedentes y elaborar el marco teórico de la investigación, es posible suponer a manera de hipótesis que la procedencia del viento para la ciudad es variable principalmente por las diferencias en la cantidad y tiempo de exposición solar según horas del día y las épocas del año, además por la ubicación geográfica y características físicas donde al ser un valle, los vientos cambian constantemente. 


\section{Enfoque}

Para obtener evidencia concisa es necesario desarrollar la investigación con un enfoque mixto; descriptivo y cuantitativo. Se basa en la observación y descripción de las gráficas de rosas de los vientos derivadas de datos estadísticos registrados por aparatos especializados.

\section{Materiales y métodos}

Para realizar el análisis que se describe en este documento se ejecutan las siguientes acciones:

1. Trabajo de documentación y análisis bibliográfico, donde principalmente se buscan los datos de interés; dirección del viento para Ciudad Valles en los distintos meses, épocas y horas.

2. Visitar oficinas regionales de la CONAGUA para consultar los procedimientos de solicitud de información pública.

3. Redacción de oficio solicitud dirigido al responsable de la CONAGUA, el Sr. Lic. Jesús Liñán Guevara en el año 2013 y posteriormente; en el año 2016, al Ing. Alejandro Vázquez Acosta.

4. Recepción y revisión de la información solicitada, la cual se recibe vía correo electrónico en formato digital; con extensión de texto (.txt) y Excel (.xls).

5. Trabajo de gabinete: utilizar todos los archivos recibidos para elaborar un concentrado de información en formato de Excel para optimizar los tiempos en generar resúmenes, tablas y en su caso la creación de gráficas y evidentemente el cálculo de resultados.

6. Revisar manual de usuario del programa WRPLOT view para conocer la forma de generar las gráficas rosas de los vientos.

7. Una vez que se organiza la información tal y como es necesaria para utilizarla en el programa WRPLOT view, lo siguiente es procesar los datos para generar las rosas de los vientos y obtener la evidencia de forma clara.

\section{Particularidades de la variable climática Viento}

El registro de la dirección del viento se realiza en base a los grados, en este sentido, es importante recordar y distinguir que los ángulos más utilizados se basan en el sistema sexagesimal, que se miden con la rotación de una línea en dirección contraria a las manecillas del reloj, comenzando la medición respecto de la horizontal. Para registrar la dirección del viento este sistema cambia; la forma correcta para el registro del ángulo de la dirección del viento es tomando la vertical como línea de partida; asignándole al extremo superior la orientación norte y un valor angular de inicio de cero grados; y comenzando a rotar en sentido horario, así pues, se llega a los 360 grados en el mismo punto de origen de cero grados con orientación norte. Este ángulo medido en sentido horario a partir de la vertical; desde el norte; se le denomina azimut.

A manera de distinguir lo descrito; el valor de $90^{\circ}$ del sistema sexagesimal; es la línea vertical que se encuentra apuntando hacia el extremo superior, mientras que para el uso correcto para el viento corresponde a la orientación norte con dos valores angulares; uno de partida que corresponde a $0^{\circ}$ y un valor de $360^{\circ}$ como valor final para cerrar la circunferencia.

Además es indispensable para un buen registro; tener en cuenta que el promedio para esta variable aplica de forma distinta; ejemplo de ello es suponer que tenemos valores de la dirección del viento registradas en donde los parámetros son 0 y 360 grados, en donde si pretendemos obtener el valor promedio, aplicamos la operación aritmética; $(0+360) / 2$; y se obtiene un valor de 180 grados, por lo tanto se grafica como una orientación Sur, en tanto que los 0 y 360 grados pertenecen a la orientación Norte. Para evitar este error en el cálculo y se logre graficar de forma correcta los sistemas trabajan con rangos de valores según el número de orientaciones de interés que se espera visualizar. Generalmente los rangos adecuados para trabajar son de mínimo ocho hasta un máximo de treinta y dos, esto con la intención de simplificar y facilitar la lectura de los gráficos. Otro aspecto importante de aclarar es que el registro de la dirección del viento en base a un ángulo; realmente indica la procedencia del viento, mas no hacia donde se dirige. 


\section{EMA: Estación Meteorológica Automática}

Los aparatos más utilizados para la medición y registro de la dirección y velocidad del viento son el anemómetro, la veleta, el anemómetro de placa, el anemómetro de sensor rotativo que implementa cazoletas, y los anemómetros o anemógrafos de tubo de presión. Para efecto de este trabajo se describe de la EMA; los instrumentos para medir las variables meteorológicas y las unidades en las que se registran; a continuación se presenta fotografía (figura 1) de la EMA que se encuentra ubicada en el municipio de Ciudad Valles.

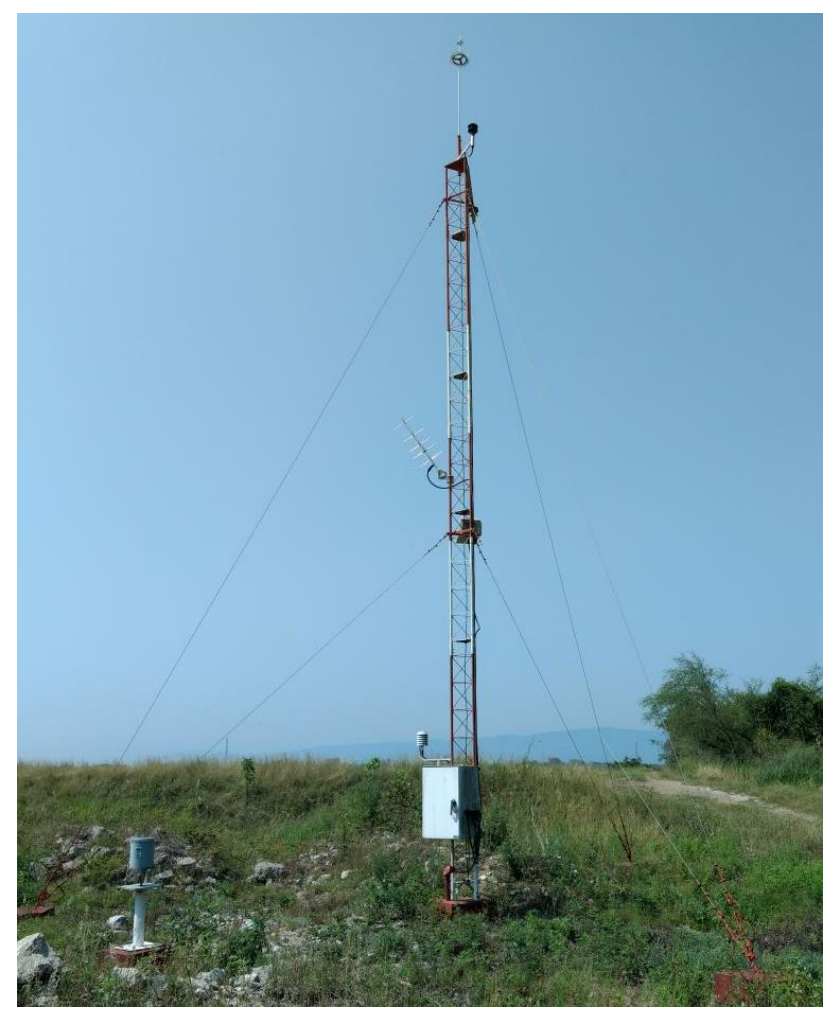

Figura 1 EMA de Ciudad Valles

Fuente: Elaboración Propia. Junio 2018

La EMA trabaja con panel solar para su consumo energético, además que son altamente resistentes a climas extremos, el respaldo de información la realiza en su disco duro para posteriormente acceder a ella o bien se les programa para que envíen la información por internet, esto implica un gran beneficio para simplificar el capital humano necesario para sus cuidados, ya que permite monitorearlas a distancia; a comparación de los observatorios meteorológicos los cuales deben contar con una persona encarga de registrar aproximadamente cada 3 horas las observaciones de las variables climáticas; estas estaciones automáticas registran en lapsos hasta de 15 minutos según la configuración que se les asigne.
Estos equipos cuentan con termómetros para medir la temperatura del aire, ubicando el termómetro dentro de una garita para protegerlo de la radiación directa y no afectar la medición debido al calentamiento por sobreexposición solar del aparato. Para la temperatura de superficie, el termómetro se sitúa a una profundidad de entre los 10 y 100 centímetros por debajo del suelo. Las unidades que se manejan son los grados centígrados; $\mathrm{C}^{\mathbf{o}}$; Kelvin; $\mathrm{K}^{\mathrm{o}}$; y Farenheit; $\mathrm{F}^{\mathrm{o}}$. Siendo la unidad internacional la de los grados Kelvin; $\mathrm{K}^{\circ}$.

Anemómetro o anemógrafo para medir la velocidad del viento; definido como el movimiento del aire en sentido horizontal; regularmente utilizando cazoletas, además de contar con veleta para registrar la dirección del viento. Las unidades más utilizadas en el caso de la velocidad son los nudos o millas náuticas; $\mathrm{Kt}$; los kilómetros recorridos en un tiempo de una hora; $\mathrm{km}-\mathrm{h}$; metros por segundo; $\mathrm{m}$-s; millas por hora; m.p.h.; y pies por segundo; ft s-1. Las unidades más utilizadas internacionalmente son los metros por segundo y los nudos.

Higrógrafo para medir la humedad del aire, siendo esta el volumen de agua; vapor; que retiene el aire presente en un metro cubico de espacio, expresada en porcentaje. Como unidades de expresión se utiliza el milibar; mbar; o hectopascal; hpa; de igual forma el kilogramo dividido entre un metro cubico; $\mathrm{kg} / \mathrm{m} 3 ; \mathrm{y}$ regularmente en porcentaje; $\%$.

Barógrafo para medir la presión atmosférica, la cual registra la presión que genera una columna de aire en una superficie de un recipiente de mercurio y se mide en las unidades de hectopascales; hPa; los cuales son más cómodos de utilizar a comparación con el pascal; un hectopascal equivale a cien pascales.

Radiógrafo para medir la energía térmica radiante; la cual se expresa en vatios por metro cuadrado; W-m2; lo que significa la cantidad de energía que recibe una superficie de un metro cuadrado, este valor tiene gran impacto en el balance de confort térmico ya que de igual forma se calcula en $\mathrm{W}$-m2, calculando la energía que recibe la persona, la que consume y la que expulsa. 
Las EMAS también se componen del pluviógrafo que sirve para medir la cantidad agua de lluvia acumulada que recibe un metro cuadrado de superficie; la cual se expresa en una medida lineal como es el caso de la altura en milímetros según el sistema internacional; $\mathrm{mm}$; esto significa que, si se expresa un registro de $1 \mathrm{~mm}$ de lluvia, representa que ha caído un litro de agua en ese metro cuadrado de superficie.

Para garantizar la correcta medición de las variables, estos aparatos se apegan a las normas mexicanas del Servicio Meteorológico Nacional; tales como la NMX-AA-166/1-SCFI2013 parte 1 y PROY-NMX-AA-166/2-SCFI2015 parte 2; Estaciones meteorológicas, climatológicas e hidrológicas. Especificaciones técnicas que deben cumplir el emplazamiento y exposición de los instrumentos de medición de las estaciones meteorológicas automáticas y convencionales.

Estas normas describen temas para el correcto manejo, calibración e instalación de los equipos, así como para su mantenimiento correctivo y preventivo. En su mayor parte cumpliendo también con los manuales y estándares de la Organización Mundial de Meteorología; OMM; organización en la que México ha tenido participación desde los años 70’s con la elaboración de diagnósticos, mapas y predicciones en materia del clima a nivel mundial.

1.9.- Aplicaciones urbanas del análisis del viento. Algunas de las aplicaciones del análisis climatológico de una ciudad, particularmente de la variable viento, es la orientación del trazo de las vialidades, donde en el caso de tener vientos con velocidades fuertes provocan túneles de viento con disconfort para el peatón, si el eje longitudinal de las vialidades están orientadas hacia la procedencia del viento, y en contraparte para las zonas con escases de viento fresco como en el caso de Ciudad Valles, esta orientación del trazo debe adecuarse al de la procedencia de los vientos. Por otra parte, en la ubicación de Zonas Industriales, ingenios y basureros municipales, la observación de la dirección y velocidad del viento es crucial para evitar la proyección de los gases, polvos y olores contaminantes hacia las zonas habitacionales. Recordar también la correcta ubicación de patios de molinos de viento para utilizarlos como generadores de energía eléctrica para la vivienda o para la iluminación de zonas exteriores.
La zona cálido húmeda a escala global generalmente se encuentra ubicada sobre la línea del ecuador y se caracteriza por recibir los rayos del sol de forma casi perpendicular, provocando constantemente temperaturas entre los 22 y $26^{\circ} \mathrm{C}$; siendo una subdivisión el clima cálido subhúmedo, este tiene un comportamiento similar, a diferencia de la cantidad de lluvia registrada, en promedio $3000 \mathrm{~mm}$ para la zona cálido húmeda contra $1500 \mathrm{~mm}$ del clima cálido subhúmedo; como es el caso de Ciudad Valles.

Ciudad Valles registra una temperatura promedio de $24.7^{\circ} \mathrm{C}$ y una Humedad relativa del $78 \%$ debido a la cercanía con la sierra madre, rica en vegetación e hidrología, la precipitación media anual es de $1,200 \mathrm{~mm}$; lo que hace que el contexto físico natural sea considerado generalmente como "Cálido Subhúmedo"; y las recomendaciones generales más importantes para adaptar los espacios abiertos con el afán de provocar cambios de temperatura y por consiguiente desplazamientos del aire son, entre otras cosas:

Controlar la radiación solar directa; con apoyo de obstrucciones; además de controlar también la temperatura radiante, difusa y la humedad relativa, además de protecciones solares, es necesario implementar ventilación mecánica dado que la velocidad del viento en la ciudad no es óptima para refrescar los espacios en los meses y periodos del día más calurosos.

Para la zona cálido subhúmeda, uno de los métodos usados para disminuir la humedad de la vivienda es elevar el desplante con ayuda de pilotes o estructura, lo que se le llama emplazamientos elevados, esto con la finalidad de optimizar el paso del viento y refrescar la mayor superficie de la vivienda.

Otra adecuación puntual para los inmuebles es la de redondear las esquinas para disminuir los cambios bruscos de la dirección del viento, orientar las ventanas pequeñas de frente al viento y colocarlas en la parte inferior de los muros para captar el aire fresco; y en contraposición las ventanas altas para darle mayor circulación de salida al aire caliente. 
El viento es también una carga especial en el cálculo estructural de edificios, y sirve para determinar la posibilidad de construir inmuebles con alturas considerables, además de permitir la selección de materiales, espesores de cristales y sistemas constructivos adecuados para las distintas intensidades; un ejemplo de ello es el caso de la ciudad de Oaxaca, donde se vio en la necesidad de sustituir la velaría del auditorio Guelaguetza por otra más resistente, esto debido a las fuertes ráfagas de viento que desprendieron la original.

Otro de los aspectos significativos de conocer las condiciones del viento, es analizar el aporte que esta variable tiene para el confort térmico de la persona en espacios exteriores para el caso del urbanismo, e interiores para la arquitectura. Según la evaluación del confort para las condiciones ambientales de Ciudad Valles, calculadas con los valores de las variables climatológicas se recomienda la ventilación mecánica en un horario de 11:00 h a 19:00 h especialmente del mes de mayo al mes de septiembre; y en horarios de 13:00 h a las 15:00 h, en el mes de abril; es necesario también incentivar el movimiento del aire. (Zapata 2017: 26)

La velocidad del viento ideal oscila entre los 2 y $4 \mathrm{~m} / \mathrm{s}$, velocidades que Ciudad Valles presenta la mayor parte del tiempo, sin embargo, estos datos son medidos en contextos distintos a las de la mancha urbana, zona donde los inmuebles obstruyen y modifican significativamente la dirección y su velocidad.

\section{Conclusiones y recomendaciones}

En base a la revisión que se realiza se logra confirmar la dirección del viento para Ciudad Valles a lo largo de los nueves años, siendo esta dirección; en caso de generalizar; noroeste y sureste, y siendo aún más específicos; noroeste y este sureste; NO y ESE. Coincidiendo con la información del periódico oficial del estado de San Luis Potosí del año 1999 en la dirección NO.

Dada la magnitud de los datos con los que se trabaja, se tiene la oportunidad de resumir la dirección y velocidad del viento para cada mes del año como se muestra en la tabla 1; considerando el periodo del año 2004 al año 2011 y posteriormente el periodo del año 2014 y 2015 ya que no se obtuvieron datos de los años 2012 y 2013.

\begin{tabular}{|l|l|r|}
\multicolumn{1}{c}{ Mes } & \multicolumn{1}{c}{ Dirección } & Velocidad $\mathbf{~} / \mathbf{s}$ \\
\hline Enero & NO, SE & 1.46 \\
\hline Febrero & ESE, NO & 1.69 \\
\hline Marzo & ESE & 2.15 \\
\hline Abril & ESE & 2.44 \\
\hline Mayo & E & 2.32 \\
\hline Junio & ENE & 2.06 \\
\hline Julio & E, NO & 1.51 \\
\hline Agosto & ESE, NO & 1.48 \\
\hline Septiembre & NO, ESE & 1.28 \\
\hline Octubre & NO, ESE & 1.34 \\
\hline Noviembre & NO, SE & 1.21 \\
\hline Diciembre & NNO, ESE & 1.30 \\
\hline
\end{tabular}

Tabla 1 Promedio de la velocidad del viento en un periodo de 9 años

Fuente: Elaboración Propia

Es importante enunciar que, si generalizamos la dirección de procedencia, estas direcciones se presentan en todos los meses del año, por lo que es necesario desglosarla en épocas y en los periodos del día para ser aún más específicos y verificar su comportamiento según los distintos escenarios estacionales, mensuales y de horario, para así garantizar un mejor análisis. En la tabla 2 se revisa la dirección y velocidad del viento por estación; en la tabla 3 se revisa la dirección y velocidad del viento según el horario del día y en la tabla 4 que se deriva de la anterior; se resume el comportamiento de ambos parámetros según el periodo del día.

\begin{tabular}{|l|l|r|}
\multicolumn{1}{|c}{ Época } & \multicolumn{1}{c}{ Dirección } & Velocidad m/s \\
\hline Primavera & E, SO, ENE & 2.50 \\
\hline Verano & E, NO & 1.60 \\
\hline Otoño & NO, ESE & 1.30 \\
\hline Invierno & ESE, NO & 1.80 \\
\hline
\end{tabular}

Tabla 2 Dirección y velocidad del viento estacional en un periodo de 9 años

Fuente: Elaboración Propia

\begin{tabular}{|l|l|r|}
\hline Horario & Dirección & Velocidad m/s \\
\hline 06:00h - 12:00h & NO & 0.70 \\
\hline 12:00h - 18:00h & NO & 1.30 \\
\hline 18:00h - 00:00h & ESE & 3.10 \\
\hline 00:00h - 06:00h & ESE & 1.90 \\
\hline
\end{tabular}

Tabla 3 Dirección y velocidad del viento por horario en un periodo de 9 años

Fuente: Elaboración Propia

\begin{tabular}{|l|l|r|}
\hline Periodo del día & Dirección & Velocidad m/s \\
\hline Mañana y Tarde & NO & 1.10 \\
\hline Noche y Madrugada & ESE & 2.40 \\
\hline
\end{tabular}

Tabla 4 Dirección y velocidad del viento por periodo del día en un periodo de 9 años

Fuente: Elaboración Propia 
De lo anterior es posible establecer entonces que en el transcurso del día; con sol; la dirección del viento es noroeste; NO; y para el momento en que se oculta el sol; en la noche; la dirección del viento es este sureste; ESE. Hasta este momento, en la opinión del investigador, la observación más importante del estudio.

Respecto de la velocidad del viento para Ciudad Valles, se tiene registrado en el rango de los 2 y 4 metros por segundo en la mayor parte del año, tomando en cuenta los colores más relevantes de las rosas de los vientos.

Los más relevante es la observación de la curva de comportamiento del viento para las distintas horas del día, donde se observa que para la mayor parte de los meses y estaciones el horario con menor velocidad de viento esta entre las 6:00h y 15:00h aproximadamente, en la estación de otoño se observa una variación ligera en la curva donde se identifica que es la temporada donde este lapso se recorre para iniciar desde la madrugada; de las 4:00h hasta las 15:00h.

Las velocidades más fuertes se alcanzan en la noche; entre las 18:00h y 00:00h con un promedio de $3.1 \mathrm{~m} / \mathrm{s}$; y en el periodo de la madrugada; entre las 00:00h y 6:00h con un promedio de $1.9 \mathrm{~m} / \mathrm{s}$. mientras que para la mañana y la tarde solo se llega a los $0.7 \mathrm{~m} / \mathrm{s}$ y 1.3 $\mathrm{m} / \mathrm{s}$ respectivamente, en los horarios de 6:00h hasta las 18:00h; horarios en que las personas y negocios normalmente desarrollan sus actividades diarias, por lo que hay que prestar importancia para dar posibles soluciones.

Como conclusión final, es importante destacar que la dirección del viento juega un papel esencial en el urbanismo bioclimático y la arquitectura; y más en el grado de confort de la persona en espacios abiertos, por lo que es necesario tener en cuenta que la diferencia en la velocidad y dirección del viento se presentará constantemente; así que se recomienda realizar; además de la observación de las normales climatológicas y las de este trabajo, un análisis del comportamiento micro climático del sitio de interés, ya que en principio estos datos pertenecen a una escala climática distinta, por lo que si bien ofrecen un parámetro bastante aceptable, son valores originados por un contexto físico inmediato.

\section{Referencias}

ÁLVAREZ, A López, (2004). Cambio climático y microclimas urbanos en ciudades del centro de cuba. Reflexiones para el planeamiento a través de sig. Argentina. Vol. 8, $\mathrm{N}^{\mathrm{o}}$ 1, 2004. Impreso en la Argentina. ISSN 0329-5184

CHICAS Molina, Juan Carlos. (2012). Morfología urbana y Clima urbano, estudio de microclimas urbanos en Santiago de Chile, mediante la aplicación del concepto de cañón urbano e índices de confort térmico. Instituto de Estudios Urbanos y Territoriales de la Pontificia Universidad. Santiago de Chile.

\begin{tabular}{lllr} 
COMITÉ DE & \multicolumn{2}{c}{ PLANEACIÓN } & DEL \\
DESARROLLO MUNICIPAL & Plan & de \\
desarrollo municipal 2012 & 2015. & H. \\
Ayuntamiento de Ciudad Valles. México. &
\end{tabular}

CONAGUA (2012). Servicio Meteorológico Nacional: 135 años de historia en México. Secretaría de Medio Ambiente y Recursos Naturales. México. ISBN: 978-607-7908-63-0

DIRECCIÓN GENERAL DE NORMAS DE LA SECRETARÍA DE ECONOMÍA. Proyecto de norma mexicana PROY-NMX-AA-166/2SCFI-2015 estaciones meteorológicas, climatológicas e hidrológicas. Parte 2: especificaciones técnicas que deben cumplir el emplazamiento y exposición de los instrumentos de medición de las estaciones meteorológicas automáticas y convencionales. Publicada en el Diario Oficial de la Federación el 11/05/2015. México.

DIRECCIÓN GENERAL DE NORMAS DE LA SECRETARÍA DE ECONOMÍA. Norma Mexicana NMX-AA-166/1-SCFI-2013 estaciones meteorológicas, climatológicas $e$ hidrológicas - parte 1: Especificaciones técnicas que deben cumplir los materiales e instrumentos de medición de las estaciones meteorológicas automáticas y convencionales. Publicada en el Diario Oficial de la Federación el 18 de julio de 2013. México.

GEIGER, R., Aron, R. H., Todhunter, P. The Climate Near The Ground, $5^{\text {a }}$ edición. Vieweg \& Sohn. Weisbaden, 1995.

GIVONI, Baruch (1998). Climate considerations in building and urban design. John Wiley \& Sons, Inc. United States of America.

ZAPATA-PADILLA, Néstor Juan, PEDRAZA-GÓMEZ, Carlos, BOJÓRQUEZ-VARGAS, Alma Rafaela, HERNÁNDEZ-GONZÁLES, Gisela Beatriz. Ciclos del viento como condicionante de diseño bioclimático y urbano para Ciudad Valles, S.L.P. Revista de Ingeniería Civil. 2019 
GOBIERNO DEL ESTADO DE SAN LUIS POTOSÍ. (1999). Periódico oficial del gobierno del estado libre y soberano de san Luis potosí de 1999. México.

GOBIERNO DEL ESTADO DE SAN LUIS POTOSÍ. (2012) Plan estatal de desarrollo urbano de San Luis Potosí 2012-2030. México.

H. AYUNTAMIENTO DE CIUDAD VALLES. Plan Municipal de Desarrollo de Ciudad Valles, San Luis Potosí 2004 - 2006. México.

H. AYUNTAMIENTO DE SAN LUIS POTOSÍ. (2003). Plan del centro de población estratégico San Luis Potosí soledad de graciano Sánchez. México.

H. AYUNTAMIENTO DE SAN LUIS POTOSÍ. (2018). El plan del centro de población estratégico San Luis Potosí soledad de graciano Sánchez. México.

HIGUERAS, Ester (2013). Urbanismo Bioclimático. (1a edición, 5a tirada) ISBN: 9788425220715

INSTITUTO NACIONAL DE ESTADÍSTICA GEOGRAFÍA E INFORMÁTICA (1988). Anuario estadístico del estado de San Luis Potosí de 1988. Gobierno del estado de San Luis Potosí. México. ISBN 968-892-098-3

\section{INSTITUTO NACIONAL DE ESTADÍSTICA} Y GEOGRAFÍA (2009). Prontuario de información geográfica municipal de los Estados Unidos Mexicanos del año 2009 Ciudad Valles, San Luis Potosí, Clave geo estadística 24013. México.

INSTITUTO NACIONAL DE ESTADÍSTICA Y GEOGRAFÍA (2016) Anuario estadístico y geográfico por entidad federativa 2016. Instituto Nacional de Estadística y Geografía. México. ISBN 978-607-739-993-3.

INSTITUTO NACIONAL DE ESTADÍSTICA Y GEOGRAFÍA (2016). Anuario estadístico y geográfico por entidad federativa 2016. México.

INSTITUTO NACIONAL DE ESTADÍSTICA Y GEOGRAFÍA (2017) Anuario estadístico y geográfico de San Luis Potosí 2017. México.
IVANEGA, Miriam. (2009). Construcciones Urbanas y Medio Ambiente. http://biblio.juridicas.unam.mx/libros/6/2735/7. pdf

LYNCH, Kevin. (2008). La imagen de la ciudad. Gustavo gili. SL, Barcelona, 1984, 1998.

MÉNDEZ, Carlos M. Análisis Estadístico de la Velocidad y Dirección del Viento en Los TaquesEstado Falcón. http://www.fglongatt.org.ve

(2009)

MONROY, Manuel Martín. (2001). Claves del Urbanismo Bioclimático. Artículo publicado en revista BASA. ULPGC Basa, ISSN 0213-0653, No. 24, 2001, págs. 148-159.

OCHOA, José Manuel y MARINCIC, Irene. (2002) Diseño Microclimático de Espacios Exteriores confortables y energéticamente eficientes. XXVI Semana Nacional de Energía Solar, Noviembre de 2002, Chetumal, México.

OKE, T.R. (1987). Boundary layer climates. (2nd ed.). London: Routledge.

ORGANIZACIÓN METEOROLÓGICA MUNDIAL (2015) Manual del Sistema de Información de la OMM Anexo VII al Reglamento Técnico de la OMM Anexo VII al Reglamento Técnico de la OMM OMM-N ${ }^{\circ} 1060$. Organización Meteorológica Mundial. ISBN 978-92-63-31060-6

SCHILLER, Silvia de. (2001). Isla de calor, Microclima Urbano y Variables de Diseño, estudios en Buenos Aires y Rio Gallegos. Argentina. ISSN 0329-5184

SECRETARÍA DE LA ORGANIZACIÓN METEOROLÓGICA MUNDIAL (2001) Directivas de orientación la enseñanza y formación profesional del personal en meteorología e hidrología operativa Volumen I: Meteorología. OMM-NO. 258, 4ta edición. Organización Meteorológica Mundial. ISBN 9263-14258-0

TRUJILLO, Carlos y OSPINA, Ricaurte y PARRA, Hernando. (2010). Arquitectura y Urbanismo Bioclimático: Presente y Futuro para el Hábitat del Hombre Contemporáneo. Scientia et Technica Año XVII, No 46, diciembre 2010. Universidad Tecnológica de Pereira. ISSN 0122-1701 revistas.utp.edu.co 
VÁZQUEZ, Gloria y RESTREPO, Alirio y NOLASCO, Quevedo y CRESPO, Guillermo y PORTOCARRERO, Adolfo. (2010). Manual Teórico Práctico del Observador Meteorológico de Superficie. Comisión Nacional del Agua CONAGUA. www.conagua.gob.mx

ZAPATA, Néstor. (2016). Diagnóstico del microambiente urbano en la avenida Miguel Hidalgo, en la zona centro de Ciudad Valles, San Luis Potosí, México. (Tesis de maestría), Facultad de Arquitectura, Diseño y Urbanismo de la Universidad Autónoma de Tamaulipas. Tampico, Tamaulipas. México.

ZAPATA, Néstor. (2017). Confort humano en microclimas urbanos (Calle Miguel Hidalgo, $C d$. Valles, SLP, MX.). TLATEMOANI Revista Académica de Investigación. No. 25, agosto 2017. Eumed.net. España. ISSN: 19899300 revista.tlatemoani@uaslp.mx 